\title{
Expression of UDP-glucuronosyltransferase 1A, nuclear factor erythroid-E2-related factor 2 and Kelch-like ECH-associated protein 1 in colonic mucosa, adenoma and adenocarcinoma tissue
}

\author{
MIN WANG ${ }^{1}$, YING-YING QI ${ }^{1}$, SHUO CHEN ${ }^{1}$, DE-FENG SUN ${ }^{2}$, SHUAI WANG ${ }^{1}$, \\ JIAN $\mathrm{CHEN}^{3}$, YAN-QING $\mathrm{LI}^{3}$, WEI HAN ${ }^{3}$ and XIAO-YUN YANG ${ }^{3}$ \\ ${ }^{1}$ Department of Geriatrics and Gastroenterology, Qi-Lu Hospital of Shandong University, Key \\ Laboratory of Proteomics of Shandong Province, Jinan, Shandong 250012; ${ }^{2}$ Department of Biochemistry \\ and Molecular Biology, Shandong Medical School, Jinan, Shandong $250002 ;{ }^{3}$ Department of \\ Gastroenterology, Qi-Lu Hospital of Shandong University, Jinan, Shandong 250012, P.R. China
}

Received February 21, 2012; Accepted May 31, 2012

DOI: $10.3892 / \mathrm{ol} .2012 .850$

\begin{abstract}
We investigated the expression of UDP-glucuronosyltransferase 1A (UGT1A), nuclear factor erythroid-E2-related factor 2 (Nrf2) and Kelch-like ECH-associated protein 1 (Keap1) in normal colonic mucosa, adenoma tissue and adenocarcinoma tissue, to analyze the correlation between their expression and the clinicopathological features of adenocarcinoma and their roles in colonic carcinogenesis. Using immunohistochemical analysis, we investigated the expression of UGT1A, Nrf2 and Keap1 in normal colonic mucosa $(n=24)$, adenoma tissue $(n=30)$ and adenocarcinoma tissue $(n=77)$. We found that the positive rate of UGT1A was $83.3 \%$ in normal colonic mucosa, $80.0 \%$ in adenoma tissue and significantly lower, $53.2 \%$, in adenocarcinoma tissue (normal colonic vs. adenoma tissue, $\mathrm{P}=0.071$; normal colonic vs. adenocarcinoma tissue, $\mathrm{P}=0.023$; adenoma vs. adenocarcinoma tissue, $\mathrm{P}=0.019)$. The expression levels of $\mathrm{Nrf} 2$ were high in adenoma and adenocarcinoma tissues, with positive rates of 70.0 and $87.0 \%$, respectively, but were significantly lower in normal colonic tissue, with a positive rate of $41.7 \%$ (normal colonic vs. adenoma tissue, $\mathrm{P}=0.000$; normal colonic vs. adenocarcinoma tissue, $\mathrm{P}=0.000$; adenoma vs. adenocarcinoma tissue, $\mathrm{P}=0.000$ ). The positive rate of Keap1 was $54.2 \%$ in normal mucosa, $70.0 \%$ in adenoma tissue and $61.0 \%$ in adenocarcinoma tissue
\end{abstract}

Correspondence to: Dr Min Wang, Department of Geriatrics and Gastroenterology, Qi-Lu Hospital of Shandong University, Key Laboratory of Proteomics of Shandong Province, 107 Wenhuaxi Road, Jinan, Shandong 250012, P.R. China

E-mail: doctorminmin@163.com

Key words: UDP-glucuronosyltransferase 1A, transcription factor, nuclear factor erythroid-E2-related factor 2, Kelch-like $\mathrm{ECH}$-associated protein 1, colonic adenoma, colonic adenocarcinoma (normal colonic mucosa vs. adenoma tissue, $\mathrm{P}=0.200$; normal colonic vs. adenocarcinoma tissue, $\mathrm{P}=0.040$; adenoma vs. adenocarcinoma, $\mathrm{P}=0.002)$. In addition, there was no correlation between the expression of $\mathrm{Nrf} 2 / \mathrm{Keap} 1$ in adenoma and adenocarcinoma tissues $(\mathrm{r}=0.067, \mathrm{P}=0.723 ; \mathrm{r}=0.042, \mathrm{P}=0.715$, respectively). The results suggest that decreased expression of UGT1A and the dysregulation of the Nrf2/Keap1 system may be related to colonic carcinogenesis.

\section{Introduction}

Colon cancer is one of the most common malignant tumors in humans, and the occurrence and development of colon cancer are closely correlated with lifestyle, genetic factors, mutations and adenoma. The consumption of heat-treated meat is a risk factor for colon cancer, as meat cooked at a high temperature may produce carcinogenic heterocyclic amines. It has been reported that heterocyclic amines can cause DNA damage in intestinal epithelial cells in patients with colon cancer (1). Uridine diphosphate glucuronyl transferase (UGT), one of the most important phase II metabolic enzymes for biotransformation, catalyzes the binding of $\mathrm{N}$-hydroxy compounds and glucuronic acid, which in turn prevents the DNA mutagenesis caused by heterocyclic amines (2). The UGT family, mainly composed of the UTG1A and UTG2B subfamilies, is expressed in the liver, gastrointestinal tract, gall bladder and breast (3-5). Certain UTG1A isoforms, including UTG1A7, UTG1A8 and UTG1A10, are mainly distributed in colonic mucosa, and exert a protective effect through the glucuronide reaction (6). A transcription factor, nuclear factor erythroid-E2-related factor 2 (Nrf2), plays a key role in the maintenance of cell redox homeostasis. Kelch-like ECH-associated protein-1 (Keap1) is a negative regulation factor of Nrf2. When the body is stimulated by chemical substances, carcinogens or oxidative stress, the Nrf2/Keap1 signaling pathway is activated and Nrf2 enters the nucleus to regulate the gene expression of detoxification enzymes. Hence, the Nrf2/Keap1 signaling pathway 
functions as a key system in response to stress stimulation. It has been suggested that the Nrf2/Keap1 pathway affects cell proliferation and the response of the body to chemotherapy in lung, head and neck, breast and pancreatic cancers $(7,8)$. In the present study, we investigated the expression of UGT1A, Nrf2 and Keap1 in colon cancer and elucidated their correlation with the clinicopathological features of patients with colon cancer.

\section{Materials and methods}

Materials. All specimens were acquired from surgical patients or patients undergoing digestive endoscopic inspection admitted to Qilu Hospital of Shandong University between March 2010 and June 2011. There were 77 patients with colon cancer, including 41 males and 36 females, who had an average age of 58.6 years. None of the patients were treated with chemotherapy, radiation therapy or other biological treatment, and none had a long-term regular medication history. All patients were pathologically diagnosed with colon cancer. There were 30 patients with colon adenoma, including 19 males and 11 females, with an average age of 56.1 years. None of the patients had a long-term regular medication history. All patients were pathologically diagnosed with colon adenoma (including tubular, villous and tubular villous adenoma based on the Morson classification). In addition, normal colonic mucosa specimens were acquired from 24 healthy subjects (confirmed by electronic colonoscopy) and volunteers (13 males and 11 females) who had an average age of 50.0 years. The major clinical medical records included gender, age, tumor grade, tumor infiltration and lymph node metastasis. All specimenrs were fixed with $4 \%$ paraformaldehyde and embedded with paraffin and slices at a 5- $\mu \mathrm{m}$ thickness were prepared. The stage and grade of colon cancer were determined by the American Joint Committee on Cancer/Union for International Cancer Control, (AJCC/UICC; Table I).

Reagents. A rabbit anti-human polyclonal antibody specific for Keap1 (1:150) was purchased from ABCAM (Cambridge, MA, USA). Rabbit anti-human polyclonal antibodies specific for Nrf2 (1:100) and UTG1A (1:150) were purchased from Santa Cruz Biotechnology, Inc. (Santa Cruz, CA, USA). The immunohistochemistry kit (PV-9001) and DAB chromogenic kit were purchased from Beijing Zhongshan Golden Bridge Biotechnology Company (Beijing, China).

Immunohistochemistry. The slices of $5 \mu \mathrm{m}$ thickness were dewaxed, hydrated and washed with PBS for 5 min, twice. The slices were immersed in a $92-98^{\circ} \mathrm{C}$ citrate buffer solution for 20-30 min and then cooled down for $30 \mathrm{~min}$. After being washed with PBS three times, the slices were treated with $3 \%$ $\mathrm{H}_{2} \mathrm{O}_{2}$ for 15 min and washed with PBS three times. The slices were incubated with primary antibodies (diluted in PBS) at $4^{\circ} \mathrm{C}$ overnight and a control group was established with the antibody replaced with PBS. The following day, the slices were incubated at $37^{\circ} \mathrm{C}$ for $30 \mathrm{~min}$ and washed with PBST three times. After being dried, the slices were treated with reagent 1 (Polymer Helper), incubated at $37^{\circ} \mathrm{C}$ for $20 \mathrm{~min}$ and washed with PBST three times. The slices were then treated with reagent 2 (poly-HRP anti-rabbit $\mathrm{IgG}$ ), incubated at $37^{\circ} \mathrm{C}$ for $20 \mathrm{~min}$ and washed with PBST three times. The slices were then stained with DAB. After being washed with water, the slices were stained with hematoxylin and washed with water. The slices were treated with $1 \% \mathrm{HCl}$-ethanol to degrade the excessive hematoxylin for a few seconds and then immersed in ammonia for $3 \mathrm{~min}$. After being washed with water twice, the slices were dehydrated and mounted with gum. The slices were observed under a light microscope and images were captured and stored on a computer for analysis.

Data analysis. The Nrf2-positive cells were determined by brown stained cytoplasm or nucleus and the Keap1- and UGT1A-positive cells were determined by brown stained cytoplasm. As described previously $(8,9)$, the ratio of positive cells and the intensity of the staining were analyzed as follows: A, score based on the ratio of stained cells $(<1 / 3$ positive cells, 1 point; $1 / 3-2 / 3,2$ points; $\geq 2 / 3,3$ points); $B$, score based on the intensity of staining (no positive cell, 0 point; pale brown, 1 point; brown, 2 points; deep brown, 3 points). The integrated score $=\mathrm{A} \times \mathrm{B}$. The final result was determined as negative if the integrated score was 0 and positive if the score was greater than or equal to 1 . All pathological and immunohistochemical analysis was performed by two pathology professors.

Statistical analysis. All data analysis was performed using SPSS 13.0 software (SPSS, Inc., Chicago, IL, USA). The data were analyzed using the Chi-square test and Spearman rank correlation analysis. $\mathrm{P}<0.05$ was considered to indicate a statistically significant result.

\section{Results}

Expression of UGT1A in normal colonic mucosa, adenoma tissue and adenocarcinoma tissue. In the normal colonic mucosa, UGT1A was distributed in the epithelial cell cytoplasm, especially the perinuclear area, with a positive rate of $83.3 \%(20 / 24)$, and the staining intensity markedly increased from the base of the crypt to the surface epithelial cells. In adenoma tissue, the positive rate of UGT1A was $80.0 \%(24 / 30)$, but the staining intensity did not markedly increase from the base of the crypt to the surface epithelial cells (Table I; Fig. 1). Comparing these two groups, we found no significant difference $(\mathrm{P}=0.071)$. In the adenocarcinoma tissue, UGT1A showed a slight expression in the cytoplasm with a significantly reduced positive rate of 53.2\% (41/77; Table I; Fig. 1). Compared with the normal colonic mucosa and the adenoma tissue, the adenocarcinoma tissue was significantly different ( $\mathrm{P}=0.023$ and $\mathrm{P}=0.019$, respectively). Of the 6 cases of negative adenoma tissue, 4 cases showed intraepithelial neoplasia at different degrees. Furthermore, the expression of UGT1A in colon cancer was not correlated with gender, age, tumor infiltration or lymph node metastasis ( $\mathrm{P}>0.05$; Table II).

Expression of Nrf2 in normal colonic mucosa, adenoma tissue and adenocarcinoma tissue. The positive rate of Nrf 2 increased from $41.7 \%$ (10/24) in normal colonic mucosa to $70.0 \%(21 / 30)$ in the adenoma tissue, and further to $87.0 \%(61 / 77)$ in the adenocarcinoma tissue. Nrf2 was expressed in the cytoplasm and nuclei of the normal colonic mucosa, but only in the cytoplasm of the adenoma tissue and the adenocarcinoma tissue (Table I; Fig. 2). The expression level of Nrf2 was significantly different among the three types of tissues (normal colonic vs. adenoma 
Table I. Expression of Nrf2, Keap1 and UGT1A in colonic tissues.

\begin{tabular}{|c|c|c|c|c|c|c|c|c|c|c|}
\hline \multirow[b]{2}{*}{ Group } & \multirow[b]{2}{*}{$\begin{array}{l}\text { Cases } \\
\text { (n) }\end{array}$} & \multicolumn{3}{|c|}{ UGT1A } & \multicolumn{3}{|c|}{ Nrf2 } & \multicolumn{3}{|c|}{ Keap1 } \\
\hline & & $\begin{array}{c}\text { No. } \\
\text { positive }\end{array}$ & $\begin{array}{l}\text { Positive } \\
\text { rate }(\%)\end{array}$ & P-value & $\begin{array}{c}\text { No. } \\
\text { positive }\end{array}$ & $\begin{array}{l}\text { Positive } \\
\text { rate }(\%)\end{array}$ & P-value & $\begin{array}{c}\text { No. } \\
\text { positive }\end{array}$ & $\begin{array}{l}\text { Positive } \\
\text { rate }(\%)\end{array}$ & P-value \\
\hline Normal colonic mucosa & 24 & 20 & 83.3 & $0.071^{\mathrm{a}}$ & 10 & 41.7 & $0.000^{\mathrm{a}}$ & 13 & 54.2 & $0.200^{\mathrm{a}}$ \\
\hline Adenoma tissue & 30 & 24 & 80.0 & $0.019^{\mathrm{b}}$ & 21 & 70.0 & $0.000^{\mathrm{b}}$ & 21 & 70.0 & $0.002^{\mathrm{b}}$ \\
\hline Adenocarcinoma tissue & 77 & 41 & 53.2 & $0.023^{\mathrm{c}}$ & 67 & 87.0 & $0.000^{c}$ & 47 & 61.0 & $0.040^{\mathrm{c}}$ \\
\hline
\end{tabular}

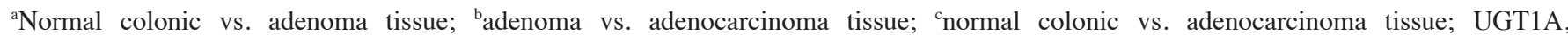
UDP-glucuronosyltransferase 1A; Nrf2, nuclear factor erythroid-E2-related factor 2; Keap1, Kelch-like ECH-associated protein 1.

Table II. Correlation between expression of Nrf2, Keap1 and UGT1A and the clinicopathological characteristics of adenocarcinoma.

\begin{tabular}{|c|c|c|c|c|c|c|c|c|c|c|}
\hline \multirow[b]{2}{*}{ Group } & \multirow[b]{2}{*}{$\begin{array}{c}\text { Cases } \\
\text { (n) }\end{array}$} & \multicolumn{3}{|c|}{ UGT1A } & \multicolumn{3}{|c|}{ Nrf2 } & \multicolumn{3}{|c|}{ Keap1 } \\
\hline & & $\begin{array}{c}\text { No. } \\
\text { positive }\end{array}$ & $\begin{array}{l}\text { Positive } \\
\text { rate }(\%)\end{array}$ & P-value & $\begin{array}{c}\text { No. } \\
\text { positive }\end{array}$ & $\begin{array}{l}\text { Positive } \\
\text { rate }(\%)\end{array}$ & P-value & $\begin{array}{c}\text { No. } \\
\text { positive }\end{array}$ & $\begin{array}{l}\text { Positive } \\
\text { rate (\%) }\end{array}$ & P-value \\
\hline Gender & & & & 0.179 & & & 0.946 & & & 0.982 \\
\hline Male & 41 & 22 & 53.7 & & 36 & 87.8 & & 25 & 61.0 & \\
\hline Female & 36 & 19 & 52.8 & & 31 & 86.1 & & 22 & 61.1 & \\
\hline Age (years) & & & & 0.692 & & & 0.693 & & & 0.433 \\
\hline$<60$ & 44 & 26 & 59.1 & & 38 & 86.4 & & 25 & 56.8 & \\
\hline$\geq 60$ & 33 & 15 & 45.5 & & 29 & 87.9 & & 22 & 66.7 & \\
\hline Differentiation & & & & 0.656 & & & 0.549 & & & 0.044 \\
\hline High/medium & 50 & 26 & 52.0 & & 44 & 88.0 & & 35 & 70.0 & \\
\hline Low & 27 & 15 & 55.6 & & 23 & 85.2 & & 12 & 44.4 & \\
\hline Tumor infiltration & & & & 0.122 & & & 0.318 & & & 0.009 \\
\hline $\mathrm{T} 1-\mathrm{T} 2$ & 33 & 13 & 39.4 & & 29 & 87.9 & & 26 & 78.8 & \\
\hline $\mathrm{T} 3-\mathrm{T} 4$ & 44 & 28 & 63.6 & & 38 & 86.4 & & 21 & 47.7 & \\
\hline Lymph node metastasis & & & & 0.157 & & & 0.197 & & & 0.275 \\
\hline N0 & 50 & 25 & 50.0 & & 42 & 84.0 & & 33 & 66.0 & \\
\hline$N x(x \geq 1)$ & 27 & 16 & 59.3 & & 25 & 92.6 & & 14 & 51.9 & \\
\hline
\end{tabular}

Nrf2, nuclear factor erythroid-E2-related factor 2; Keap1, Kelch-like ECH-associated protein 1; UGT1A, UDP-glucuronosyltransferase 1A.

tissue, $\mathrm{P}=0.000$; normal colonic vs. adenocarcinoma tissue, $\mathrm{P}=0.000$; adenoma vs. adenocarcinoma tissue, $\mathrm{P}=0.000)$. The expression of Nrf2 in tissues with different degrees of tumor differentiation (high/medium vs. low differentiation) or infiltration (T1+T2 vs. T3+T4) did not show any significant difference ( $\mathrm{P}>0.05$; Table II). However, Nrf2 expression was detected in the vascular muscle cells of tumors, and the expression level increased from the mucosa of the cancer tissue to the muscle tissue layer. In the adenocarcinoma tissue, the expression level of Nrf2 was not correlated with age or gender (Table II).

Expression of Keapl in normal colonic mucosa, adenoma and adenocarcinoma tissue. In the three types of tissues, Keap1 was only expressed in the cytoplasm, with a granular distribution (Fig. 3). The positive rate of Keap1 was 54.2\% (13/24) in normal colonic mucosa and $70.0 \%(21 / 30)$ in the adenoma tissue, and no significant difference was found between the two tissues $(\mathrm{P}=0.200)$. In the 77 cases of adenocarcinoma tissue, 47 showed positive Keap1 expression, leading to a positive rate of $61.0 \%$. The expression of Keap1 was significantly different among the tissues with different degrees of tumor differentiation or infiltration $(\mathrm{P}<0.05$; Table II $)$. Compared with the normal colonic mucosa and the adenoma tissue, the adenocarcinoma tissue showed a significant difference in the expression level of Keap1 (normal colonic mucosa vs. adenoma tissue, $\mathrm{P}=0.200$; normal colonic vs. adenocarcinoma tissue, $\mathrm{P}=0.040$; adenoma vs. adenocarcinoma, $\mathrm{P}=0.002$ ).

Correlation between the expression of Nrf2 and Keapl in normal colonic mucosa, adenoma tissue and adenocarci- 

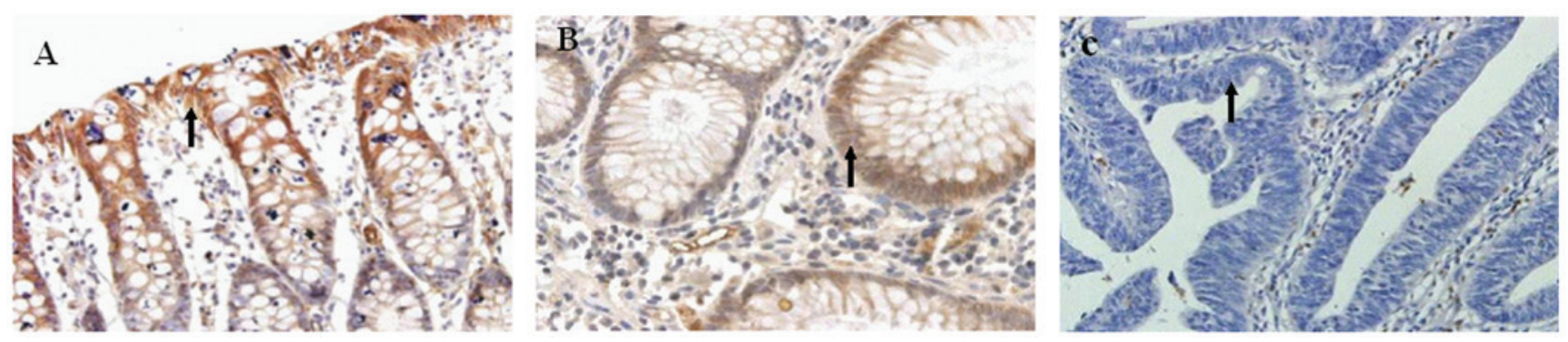

Figure 1. (A) Normal colonic tissue showing the increasing intensity of the staining from the base of the crypt to the surface epithelial cells. Positive staining was detected in the epithelial cells excluding the nuclei. (B) UGT1A was expressed in the epithelial cells excluding the nuclei in adenoma tissue. (C) UGT1A was not expressed in adenocarcinoma tissue. x400 magnification. UGT1A, UDP-glucuronosyltransferase 1A.
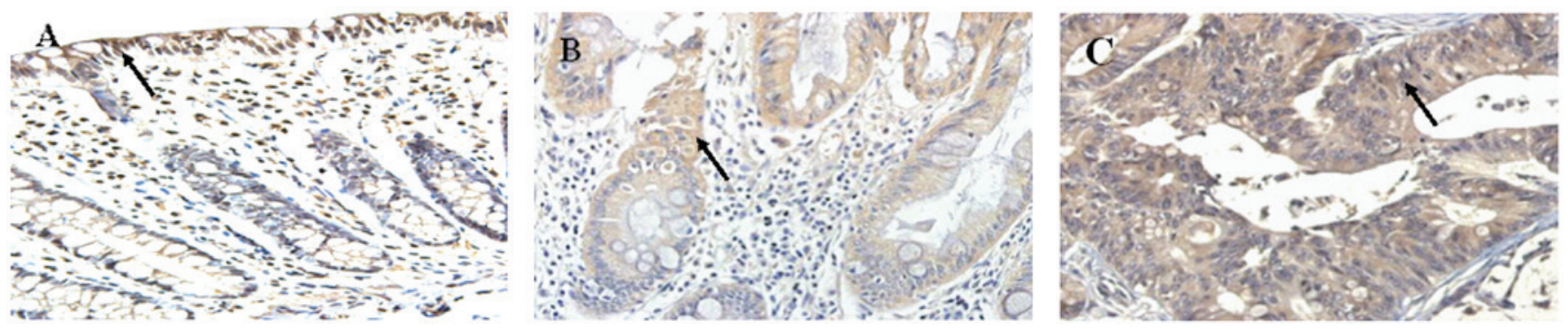

Figure 2. (A) Nrf2 was expressed in the cytoplasm and nucleus of normal colonic tissues. (B) The adenoma tissues showed strong Nrf2 expression only in the cytoplasm. (C) Strong Nrf2 staining was detected in the adenocarcinoma tissue. x400 magnification. Nrf2, nuclear factor erythroid-E2-related factor 2.
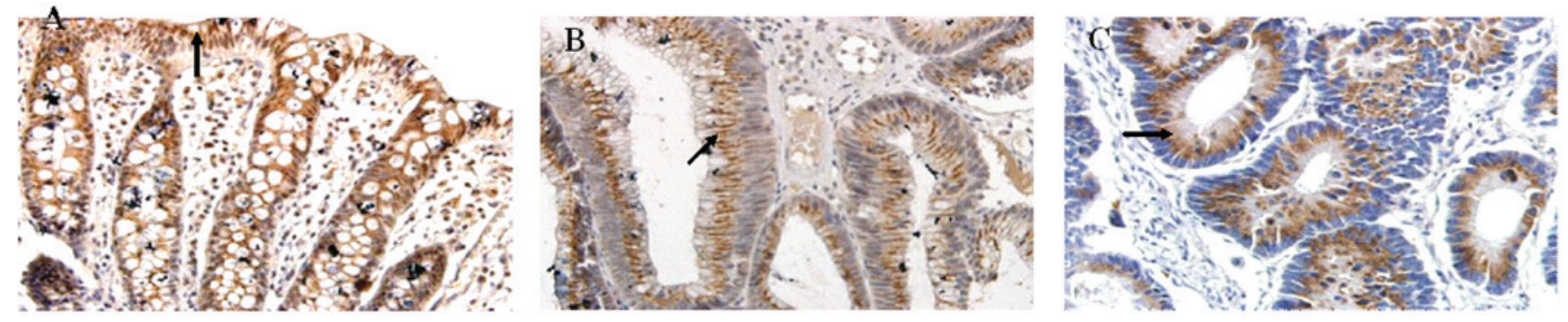

Figure 3. Keap1 staining was granular in the cytoplasm. (A) Keap1 was expressed in the cytoplasm in normal colonic tissues. (B) The adenoma tissues showed strong Keap1 expression. (C) The adenocarcinoma tissues showed strong Keap1 staining. x400 magnification. Keap1, Kelch-like ECH-associated protein 1.

noma tissue. The expression levels of Keap1 and Nrf2 were significantly correlated in normal colonic mucosa $(r=0.583$, $\mathrm{P}=0.003)$, but they were not correlated in either the adenoma $(\mathrm{r}=0.067, \mathrm{P}=0.723)$ or the adenocarcinoma tissues $(\mathrm{r}=0.042$, $\mathrm{P}=0.715)$.

\section{Discussion}

The binding reaction of N-hydroxy compounds and glucuronic acid is common in vivo, and significantly affects the metabolism of numerous drugs, toxins and carcinogens. UGT1A degrades the majority of toxins and carcinogens (10). Amines in epithelial cells may be further activated to form DNA adducts, which in turn activate the carcinogenic process by gene mutation. We found that UGT1A was highly and continuously expressed in normal colonic mucosa, while it had low or no expression in the adenoma and adenocarcinoma tissues with intraepithelial neoplasia. These observations indicate that UGT1A has a significant inhibitory effect in the early stage of colonic malignant transformation. The results of our previous study suggested that the protein and mRNA expression of UGT1A and its isoforms were decreased in adenocarcinoma tissue (11). In the present study, we found that although the UGT1A expression level was similar in normal colonic mucosa and adenoma tissue, 4 of the 6 cases of UGT1A-negative adenoma tissue showed intraepithelial neoplasia. We further found that the expression of UGT1A was decreased in the adenocarcinoma tissue. It has been suggested that UGT1A has only a slight, if any, expression level in the liver tissues with colon cancer metastases and the lymph nodes, indicating that the downregulation of UGT1A is critical for the development and infiltration of tumors (12). In the present study, we investigated the distribution of UGT1A 
using the immunohistochemical approach, which indicates the detoxification function of UGT1A. We found that UGT1A was mainly distributed in the crypt epithelium and the endometrial epithelial cells of glandular organs. As the amine carcinogens in the body are usually in contact with the intestinal mucosa, the high expression level of UGT1A in the intestinal cells acts as a chemical barrier against the carcinogenic molecules. However, UGT1A did not show an marked distribution pattern in adenoma tissues. Our previous study (13) suggested that UGT1A and its isoforms had lower catalytic activities compared with normal colonic mucosa and the UGT1A8 mutant was detected in colon cancer tissues. We thus proposed that UGT1A had a lower activity and an abnormal expression level and was unable to degrade the toxic substances quickly, which in turn prolonged the contact of toxic molecules with the mucosa to increase the risk of cancer occurrence.

Nrf2 is an important transcription factor which regulates the activity of phase II metabolic enzymes and plays a significant role in protecting cells against the oxidative stress caused by electrophilic substances and reactive oxygen species (14-16). Our results indicate that Nrf2 was mostly distributed in the nuclei, not the cytoplasm, in the normal colonic mucosa, while the opposite was found for the adenocarcinoma tissue. This was probably caused by gene mutation. A previous study on squamous cell carcinoma suggested that the gene mutation rate of Nrf2 was approximately $95.5 \%$ (17). In the adenocarcinoma tissue, the high expression of Nrf2 in the cytoplasm probably represented the overexpression of Nrf2 mutants, which resulted in the abnormal nuclear translocation and the less effective activation of phase II detoxifying enzyme gene expression, and subsequently promoted the occurrence of carcinogenesis. Our previous study on colon cancer Caco-2 cells suggested that the plant chemical sulforaphane promotes the nuclear translocation of Nrf2, while in the control group, Nrf2 was mostly located in the cytoplasm (18). This was likely caused by the Nrf 2 gene mutations induced by oxidative stress or carcinogenic damage. In the present study, we found that Nrf2 was expressed in the vascular muscle cells of tumors, and that the expression level increased from the mucosa of the cancer tissue to the muscle tissue layer, indicating that $\mathrm{Nrf} 2$ is involved in cancer invasion and metastasis. It has been previously suggested that Nrf2 is involved in the invasion and metastasis of gastric cancer (19).

Usually, the expression of Nrf2 is regulated by its negative regulating factor, Keap1, to maintain a stable low expression level. We found that Keap1 was highly expressed in the adenoma and adenocarcinoma tissues, and its expression level was correlated with the infiltration and differentiation of adenocarcinoma, indicating that Keap1 is involved in the development of cancer. The expression levels of Nrf2 and Keap1 were correlated in normal colonic mucosa, but not in the adenoma or adenocarcinoma tissues. These results indicate that the imbalance in their expression may be involved in the development of cancer. In this case, the imbalance may be caused by the upregulated expression of proteins that compete for the binding sites of Nrf2/Keap1, including sequestosome-1 and prorhymosin- $\alpha$ (20-22), which results in the slower degradation of Keap1 and the overexpression of Nrf2. It has been suggested that HNE modifies Keap1, allowing Nrf2 to enter the nucleus to take effect $(23,24)$. Future cellular studies are required to elucidate the effects of abnormal regulation and expression of the Nrf2/Keap1 pathway on the development of cancer. Changes in the expression of UGT1A, Nrf2, and Keap1 may be used as early indicators for the development of cancer to facilitate cancer intervention therapy on a molecular basis.

\section{Acknowledgements}

This study was supported by grants from Science Bonus Funds for Young Scientists of Shandong Province (No. 2007BS03017) and Natural Science Foundation of Shandong Province (No. Y2008C115).

\section{References}

1. Daniel T. S , Lisa M. G , Barbara S, et al: Inhibition of fried meat-induced colorectal DNA damage and altered systemic genotoxicity in humans by crucifera, chlorophyllin, and yogurt. PLoS One 64: 1-11, 2011.

2. Malfatti MA and Felton JS: N-glucuronidation of 2-amino-1methyl-6-phenylimidazo[4,5-b]pyridine (PhIP) and N-hydroxyPhIP by specific human UDP-glucuronosyltransferases. Carcinogenesis 22: 1087-1093, 2001.

3. Giuliani L, Gazzanga P, Caporuscio F, et al: Can down-regulation of UDP-glucuronosyltransferases in the urinary bladder tissue impact the risk of chemical cacinogenesis? Int J Cancer 91: 141-143, 2001.

4. McDonnell WM, Hitomi E and Askari FK: Identification of bilirubin UDP-GTs in the human alimentary tract in accordance with the gut as a putative metabolic organ. Biochem Pharmacol 51: 483-488, 1996.

5. Gestl SA, Green MD, Shearer DA, et al: Expression of UGT2B7, a UDP-glucuronosyltransferase implicated in the metabolism of 4-hydroxyestrone and all-trans retinoic acid, in normal human breast parenchyma and in invasive and in situ breast cancers. Am J Pathol 160: 1467-1479, 2002.

6. Strassburg CP,Nguyen N, Manns MP and Tukey RH: Polymorphic expression of UDP-glucuronosyltransferases UGT1A gene locus in human gastric epithelium. Mol Pharmacol 54: 647-654, 1998.

7. Solis LM, Behrens C, Dong W, et al: Nrf2 and Keap1 abnormalities in non-small cell lung carcinoma and association with clinicopathologic features. Clin Cancer Res 16: 3743-3753, 2010.

8. Lister A, Nedjadi T, Kitteringham NR, et al: Nrf2 is overexpressed in pancreatic cancer: implications for cell proliferation and therapy. Mol Cancer 10: 37, 2011.

9. Dango S, Sienel W, Schreiber M, et al: Elevated expression of carcinoembryonic antigen-related cell adhesion molecule 1 (CEACAM-1) is associated with increased angiogenic potential in non-small-cell lung cancer. Lung Cancer 60: 426-433, 2008.

10. Miners J, McKinnon RA and Mackenzie PI: Genetic polymorphisms of UDP-glucuronosyltransferases and their functional significance. Toxicology 181: 453-456, 2002.

11. Wang M, Li YQ, Sun DF, et al: Polymorphic expression of UDP-glucuronosyltransferase UGT1A gene locus in human colorectal epithelium. Zhongguo Bing Li Sheng Li Xue Hui 21: 1315-1320, 2005.

12. Giuliani L, Ciotti M, Stoppacciaro A, et al: UDP-glucuronosyltransferases $1 \mathrm{~A}$ expression in human urinary bladder and colon cancer by immunohistochemistry. Oncol Rep 13: 185-191, 2005.

13. Wang M, Sun DF, Li YQ, et a1: Polymorphism of UDPglucuronosyltransferase UGT1A8 gene in human colorectal cancer. Shi Jie Hua Ren Xiao Hua Za Zhi 13: 1819-1823, 2005.

14. Chan K, Han XD and Kan YW: An important function of Nrf2 in combating oxidative stress: detoxification of acetaminophen. Proc Natl Acad Sci USA 98: 4611-4616, 2001.

15. Rangasamy T, Cho CY, Thimmulappa RK, et al: Genetic ablation of Nrf2 enhances susceptibility to cigarette smoke-induced emphysema in mice. J Clin Invest 114: 1248-1259, 2004.

16. Ramos-Gomez M, Kwak MK, Dolan PM, et al: Sensitivity to carcinogenesis is increased and chemoprotective efficacy of enzyme inducers is lost in nrf2 transcription factor-deficient mice. Proc Natl Acad Sci USA 98: 3410-3415, 2001.

17. Kim YR, Oh JE, Kim MS, et al: Oncogenic NRF2 mutations in squamous cell carcinomas of oesophagus and skin. J Pathol 220: 446-451, 2010. 
18. Wang M, Li YQ, Zhong N, et al: Induction of uridine 5 'diphosphate-glucuronosyltransferase gene expression by sulforaphane and its mechanism: experimental study in human colon cancer cells. Zhonghua Yi Xue Za Zhi 85: 819-824, 2005.

19. Wang HB, Zhou CJ, Song SZ, et al: Evaluation of Nrf2 and IGF-1 expression in benign, premalignant and malignant gastric lesions. Pathol Res Pract 207: 169-173, 2011.

20. Copple IM, Lister A, Obeng AD, et al: Physical and functional interaction of sequestosome 1 with Keap1 regulates the Keap1-Nrf2 cell defense pathway. J Biol Chem 285: 16782-16788, 2010.

21. Komatsu M, Kurokawa H, Waguri S, et al: The selective autophagy substrate p62 activates the stress responsive transcription factor Nrf2 through inactivation of Keap1. Nat Cell Biol 12: 213-223, 2010.
22. Karapetian RN, Evstafieva AG, Abaeva IS, et al: Nuclear oncoprotein prothymosin alpha is a partner of Keap1: implications for expression of oxidative stress-protecting genes. Mol Cell Biol 25: 1089-1099, 2005.

23. Mahaffey CM, Zhang H, Rinna A, et al: Multidrug-resistant protein-3 gene regulation by the transcription factor Nrf2 in human bronchial epithelial and non-small-cell lung carcinoma. Free Radic Biol Med 46: 1650-1657, 2009.

24. Piccirillo S, Filomeni G, Brüne B, et al: Redox mechanisms involved in the selective activation of Nrf2-mediated resistance versus p53-dependent apoptosis in adenocarcinoma cells. J Biol Chem 284: 27721-27733, 2009. 